\title{
Influence of Correction Factor on Nearest Neighbour Hopping Parameterin Energy Dispersion Relation of Graphene Nanoribbon
}

\section{Asif Hassan* and Md Sohel Rana}

Khulna University of Engineering and Technology, Bangladesh

\begin{abstract}
We investigated on energy dispersion relation (E-KR) of graphenenanoribbon (GNR) considering its two prototypical shapes but only of AGNR in nearest neighbor interactions. Two parts $\frac{\Delta}{2}$ and $\left(\hbar \gamma_{s}\right)^{2}$ of E-KR relation have different characteristics independently expresses their importance. A correction factor $\ddot{A}_{\tilde{a}_{1}}$ is used for hopping between two edge carbon atoms to count edge relaxation. Influence of this factor on hopping parameter exemplifies the edge bond relaxation effect in AGNR.
\end{abstract}

Keywords: Energy dispersion relation; Graphene nanoribbon; Nearest neighbor interactions; Ballistic performance; Correction factor, Edge bond relaxation

\section{Introduction}

Carbon nanomaterial reveals different outstanding properties subject to their geometrical structures. Nanoscale strips of single layer graphene named as GNRs have recently been the focus on widespread efforts in theoretical and practical field [1]. There is a confinement of carriers in two directions so GNRs are a one dimension (1D) structure with [2] GNRs are expected to have electronic properties as like as in those of Carbon nanotubes (CNTs) that can be unwrapped into GNRs as there is structural similarity with compared to CNTs and effects in quantum confinement [3]. Today's theoretical statistics indicates that characteristics such like metallic or semiconducting behavior in GNRs unlike from that in CNTs $[4,5]$. Two prototypical edge shapes are formed by cutting graphene sheet along a straight line named as the armchair edge and the zigzag edge between the two edge orientations with a difference of $30^{\circ}$ in the axial direction [6]. Either one of these two "ideal" shapes or more complex geometries composed of a mixture of armchair and zigzag shaped fragments is formed depending on the cutting direction, the edges of the GNRs [7]. GNRs are classified by the number of dimer lines (zigzag lines) named as armchair (zigzag) edges on both sides across the ribbon width [8,9]. GNRs are semiconducting due to the edge effects for all sub-10 nm make more attractive for electronic device applications [10]. The electronic band structure of AGNRs are found to play an important role in the edge bond relaxation and the $3 \mathrm{NN}$ interactions [11-14] that are not prominent in CNTs. Using the NEGF formalism atomistic simulations of GNRFETs have also been described [15-18]. But they are expensive so we have examined by MATLAB simulation of the influence of correction factor on nearest neighbor of hopping parameter in AGNR. GNRs semiconducting properties can be guessed through its geometrical nature where width is [19-22] rely on $\mathrm{N}[21,23]$ in which this properties occur in GNRs when $\mathrm{m}=3 \mathrm{p}$ or $\mathrm{m}=3 \mathrm{p}+1$, where $\mathrm{p}$ is an integer [24]. At first we have calculated the main energy dispersion than without considering the correction factor we measured the energy dispersion from which we calculate the percentage of deviation or error on energy.

\section{Approach}

With a view to following the standard GNR literature convention - "armchair" and - "zigzag" denote to the shape of the edge in the transport direction of the GNR and, that is contrasting to the CNT convention [25]. An armchair ribbon is cut so that the edge looks as if it consists of repeated armchairs. The width of an armchair ribbon can be defined in terms of the number of dimer lines $(\mathrm{N})$ : [26]

$$
W_{a c}=(\mathrm{m}-1) \frac{\sqrt{3}}{2} \mathrm{a}
$$

Again we can write from the above equation

$$
\mathrm{m}=\frac{2 w_{a c}+2 \sqrt{3} a}{\sqrt{3} a}
$$

Where $a=1.42$ the nearest neighbor distance and $m=3 p$ or $m=3 p+1$ or $\mathrm{m}=3 \mathrm{p}+2$.

Now to observe the ballistic performance along with the band structures in the energy range of interest of AGNR where

E-k dispersion parameter is given by [10]:

$$
\frac{\Delta}{2}=\tilde{\mathrm{a}}_{1}\left(2 \mathrm{~s} \cos \frac{p \pi}{m+1}+1\right)+\tilde{\mathrm{a}}_{3}\left(2 \mathrm{~s} \cos \frac{2 p \pi}{m+1}+1\right)+4\left(\frac{\tilde{\mathrm{a}}_{1}+\Delta_{\gamma_{1}}}{m+1}\right) \sin ^{2} \frac{p \pi}{m+1}
$$

Where $\mathrm{p}=\mathrm{m} / 3$ or $(\mathrm{m}-1) / 3$ or $(\mathrm{m}-2) / 3$ and $\mathrm{s}=0.129$ indicates overlapping integral. [10]

Here for convenient to understand say $m=3 p$ or $3 p+1 o r 3 p+2$. Also $\left(\hbar \gamma_{s}\right)^{2}=(3 d)^{2}\left\{\begin{array}{l}-\frac{1}{2} \gamma_{1} s \cos \frac{p \pi}{m+1} \times\left[\gamma_{1}+\gamma_{3}\left(2 \cos \frac{2 p \pi}{m+1}+1\right)+4\left(\frac{\gamma_{3}+\Delta_{\gamma_{1}}}{m+1}\right) \sin ^{2} \frac{p \pi}{m+1}\right] \\ -\gamma_{3}\left(\gamma_{1}+2 \gamma_{3} \cos \frac{2 p \pi}{m+1}+4\left(\frac{\gamma_{3}+\Delta_{\gamma_{1}}}{m+1}\right) \sin ^{2} \frac{p \pi}{m+1}\right)\end{array}\right\}$ Where $\gamma_{1}=-3.2 \mathrm{eV}$ indicates first nearest neighbor hopping parameters,

*Corresponding author: Asif Hassan, Khulna University of Engineering and Technology, Khulna-9203, Bangladesh, E-mail: hassanstrong_08@hotmail.com

Received August 25, 2013; Accepted September 28, 2013; Published September 30,2013

Citation: Hassan A, Rana MS (2013) Influence of Correction Factor on Nearest Neighbour Hopping Parameterin Energy Dispersion Relation of Graphene Nanoribbon. J Nanomed Nanotechnol 4: 180. doi:10.4172/2157-7439.1000180

Copyright: $\odot 2013$ Hassan A, et al. This is an open-access article distributed under the terms of the Creative Commons Attribution License, which permits unrestricted use, distribution, and reproduction in any medium, provided the original author and source are credited. 
Citation: Hassan A, Rana MS (2013) Influence of Correction Factor on Nearest Neighbour Hopping Parameterin Energy Dispersion Relation of Graphene Nanoribbon. J Nanomed Nanotechnol 4: 180. doi:10.4172/2157-7439.1000180

Page 2 of 3

$\gamma_{3}=-0.3 \mathrm{eV}$ third nearest neighbor hopping parameters and $\Delta_{\gamma_{1}}=-0.2$ $\mathrm{eV}$ is the correction of $\gamma_{1}$ for the bonds due to the edge bond relaxation and $\mathrm{h}$ is the reduced Planck constant [11].

The main goal of our investigation is the 'term' which causes an error of correction factor in energy dispersion relation is

$$
\begin{aligned}
& \frac{\Delta_{e}}{2}=\gamma_{1}\left(2 s \cos \frac{p \pi}{m+1}+1\right)+\gamma_{3}\left(2 s \cos \frac{2 p \pi}{m+1}+1\right) \\
& \left(\hbar \gamma_{s}\right)^{2}=(3 d)^{2}\left\{-\frac{1}{2} \gamma_{1} s \cos \frac{p \pi}{m+1} \times\left[\gamma_{1}+\gamma_{3}\left(2 \cos \frac{2 p \pi}{m+1}+1\right)\right]-\gamma_{3}\left(\gamma_{1}+2 \gamma_{3} \cos \frac{2 p \pi}{m+1}\right)\right\}
\end{aligned}
$$

So the final energy dispersion equation become which has \pm value denoted as [10]

$$
\begin{aligned}
& \mathrm{E}=\sqrt{\left(\left(\frac{\Delta}{2}\right)^{2}-\left(\hbar \gamma_{s} k\right)^{2}\right)} \\
& \mathrm{E}_{e}=\sqrt{\left(\left(\frac{\Delta_{e}}{2}\right)^{2}-\left(\hbar \gamma_{s} k\right)^{2}\right)} \\
& \mathrm{E}_{e}=\sqrt{\left(\left(\frac{\Delta}{2}\right)^{2}-\left(\hbar \gamma_{s} k\right)^{2}{ }_{e}\right)} \\
& \mathrm{E}_{e}=\sqrt{\left(\left(\frac{\Delta_{e}}{2}\right)^{2}-\left(\hbar \gamma_{s} k\right)^{2}{ }_{e}\right)}
\end{aligned}
$$

To check the influence of correction factor of $\gamma 1$ we have omitted the $\Delta_{c e}$ term from equation (3), (4) for this corresponding value of $\mathrm{E}$ is obtained as $E_{e}$. Based on this value the percentage of error can be calculated.

$$
e_{r}=\frac{\text { actual value of } \left.\mathrm{E} \text { - error value of } \mathrm{E}\left(\mathrm{E}_{\mathrm{e}}\right)\right)}{\text { actual value of } E} \times 100 \%
$$

\section{Results and Discussion}

The energy dispersion relation is the exact solution of observing the edge bond relaxation of AGNR which is obtained for varying with $\mathrm{k}$ state of energy band.

We have seen that $-39.8694 \mathrm{i},-39.8695 \mathrm{i},-39.8696 \mathrm{i} \mathrm{eV}$ energy is found for successively $m=3 p, 3 p+1,3 p+2$ values where width of our device is $\mathrm{w}=2.46 \mathrm{~nm}$. Again real value of $\mathrm{E}$ is observed in -1.8153 , $-1.8153,-1.8154 \mathrm{eV}$ and then after three values complex energy value is found.

Now our main objectives is on \% of error value says that how the correction factor influence on energy dispersion in nearest neighbor hopping parameters.

The deviation is negligible as the minimum \% of error is fixed and maximum $\%$ of error is getting decreased.

\section{Conclusion}

In this paper, we have neglected the correction factor but we avoid totally the $\Delta_{c e}$ term in equation (3), (4) which results error although in nanoscale especially for AGNR not sensitive. So the value is changed after four faction point. However we have tried to demonstrate the response of correction factor on nearest neighbor hopping parameters which indicates the edge bond relaxation in AGNR at nanoscale which can be neglected as the $\%$ of error also found after four fraction point but in large scale device it is more sensitive.

\section{Acknowledgement}

The author would like to prostrate in worship to ubiquitous God, who gave me mercy to complete the paper. I would like to manifest my heartfelt thanks \& gratitude to my partner. Finally, the last but not least, I would like to thank my friends for providing continuous inspiration for the completion of the paper.

\section{References}

1. Wakabayashi k, Fujita M, Ajiki H, Sigrist M (1999) Electronic and magnetic properties of nanographite ribbons. Phys Rev B 59: 8271-8282.

2. Nakada K, Fujita M, Dresselhaus G, Dresselhaus MS (1996) Edge State in graphene ribbons: Nanometer size effect and edge shape dependence. Phys Rev B 54: 17954-17961.

3. Barone V, Hod O, Scusera GE (2006) Electron structure and stability of semiconducting grapheme nanoribbons. Nano Lett 6: 2748-2754.

4. Lee YL, Lee YW (2002) Ground state of graphite ribbons with zigzag edges. Phys Rev B 66: 245402-245406.

5. Miyamoto Y, Nakada K, Fujita M (1999) First-principles study of edge states of H-terminated graphitic ribbons. Phys Rev B 59: 9858-9861.

6. Wang $\mathrm{X}$, Ouyang $\mathrm{Y}$, Li H, Wang $\mathrm{H}$, Guo J, et al. (2008) Room-Temperature All-Semiconducting Sub-10-nm Graphene Nanoribbon Field-Effect Transistors. Phys Rev Lett 100: 206803-206807.

7. Dubois SMM, Zanolli Z, Declerck X, and Charlier JC (2009) Electronic properties and quantum transport in Graphene-based nanostructures. Eur Phys J B 72: 1-24.

8. Ritter KA, Lyding JW (2009) The influence of edge structure on the electronic properties of graphene quantum dots and nanoribbons. Nat Mater 8: 235-242.

9. Liu Z, Suenaga K, Harris PJF, lijima S (2009) Open and closed edges of graphene layers. Phys Rev Lett 102: 015501.

10. Zhao P, Choudhury M, Mohanram K, Guo J (2008) Computational Model of Edge Effects in Graphene Nanoribbon Transistors. Nano Res 1: 395-402.

11. Gunlycke D, White CT (2008) Tight-binding energy dispersions of arm chairedge graphene nanostrips. Phys Rev B 77: 115116.

12. Son YW, Cohen M, Louie S (2006) Energy gaps in graphene nanoribbons. Phys Rev Lett 97: 216803.

13. White CT, Li J, Gunlycke D, Mintmire JW (2007) Hidden one-electron interactions in carbon nanotubes revealed in graphene nanostrips. Nano Let 7: 825-830.

14. Sasaki K, Murakami S, Saito R (2005) Stabilization mechanism of edge states in graphene. Appl Phys Lett 88: 113110.

15. Fiori G, lannaccone G (2007) Simulation of graphene nanoribbon field effect transistors. IEEE Electr Device L 28: 760-762.

16. Ouyang Y, Yoon Y, Guo J (2007) Scaling behaviors of grapheme nanoribbon FETs: A three-dimensional quantum simulation study. IEEE T Electron Dev 54: 2223-2231.

17. Liang GC, Neophytou N, Lundstrom M, Nikonov DE (2007) Ballistic grapheme nanoribbon metal oxide semiconductor field-effect transistors: A full real-space quantum transport simulation. J Appl Phys 102: 054307.

18. Guan X, Zhang M, Liu Q, Yu Z (2007) Simulation investigation of double-gate CNR MOSFETs with a fully self-consistent NEGF and TB method. IEDM Tech Dig 761-764.

19. Choudhury M, Yoon Y, Guo J, Mohanram K (2008) Technology Exploration for Graphene Nanoribbon FETs. Design Automation Conference, DAC $45^{\text {th }}$ ACM/ IEEE 272-277.

20. Wang ZF, Li Q, Shi QW, Wang X, Yang J, et al. (2008) Chiral Selective Tunneling Induced Negative Differential Resistance in Zigzag Graphene Nanoribbon: A theoretical study. Apl Phy Lett 92: 133114.

21. Guo J, Yoon Y, Ouyang (2007) Gate Electrostatics and Quantum Capacitance of Graphene Nanoribbons. Nano Lett 1935-1940.

22. Unluer D, Tseng F, Ghosh W, Stan M (2009) Atomistic Deconstructionof Clear Performance Advantages of a Monolithically Patterned Wide-Narrow-Wide AllGraphene FET. Device Research Conference 75-76. 
Citation: Hassan A, Rana MS (2013) Influence of Correction Factor on Nearest Neighbour Hopping Parameterin Energy Dispersion Relation of Graphene Nanoribbon. J Nanomed Nanotechnol 4: 180. doi:10.4172/2157-7439.1000180

Page 3 of 3

23. Dietl P (2009) Numerical Studies of Electronic Transport Through Graphene Nanoribbons with Disorder. Diploma Thesis 1-76.

24. Zhao P, Choudhury M, Mohanram K, Guo J (2008) Computational Model of Edge Effects in Graphene Nanoribbon Transistors. Nano Res 1: 395-402.
25. Stan MR, Unluer D, Ghosh A, and Tseng F (2009) Graphene Devices, Interconnect and Circuits Challenges and Opportunities. IEEE Electron Device Letters 978: 69-72.

26. Geim AK, Novoselov NV (2007) The rise of graphene. Nat Mater 6: 183-191. 